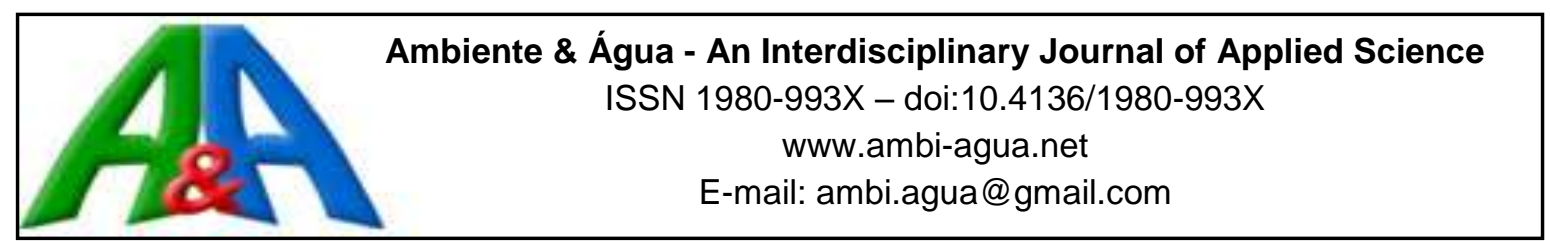

\title{
Influência topo-edafo-climática na vegetação de um fragmento de Mata Atlântica na Serra da Mantiqueira, MG
}

\author{
doi:10.4136/ambi-agua.1705
}

Received: 16 Jul. 2015; Accepted: 01 Oct. 2015

\author{
Marcela de Castro Nunes Santos Terra ${ }^{1 *}$; José Marcio de Mello ${ }^{1}$; \\ Carlos Rogério de Mello²; Rubens Manoel dos Santos ${ }^{1}$; \\ Amanda Candida Ribeiro Nunes ${ }^{1}$; \\ Marcel Régis Raimundo ${ }^{1}$ \\ Universidade Federal de Lavras (UFLA), Lavras, MG, Brasil \\ ${ }^{\mathbf{1}}$ Departamento de Ciências Florestais \\ ${ }^{2}$ Departamento de Engenharia, Setor de Água e Solo \\ *Autor correspondente: e-mail: marcelacns@gmail.com, \\ josemarcio@dcf.ufla.br, crmello@deg.ufla.br, rubensmanoel@dcf.ufla.br, \\ amandacrn@gmail.com, marcelufla@gmail.com
}

\section{RESUMO}

Compreender as correlações existentes entre variáveis ambientais e a distribuição das espécies em uma floresta, sua dinâmica e estoque de carbono é um dos principais objetivos em ecologia florestal. Nesse sentido o presente trabalho objetivou correlacionar aspectos da vegetação com variáveis topo-edafo-climáticas em um remanescente florestal em Bocaina de Minas, Serra da Mantiqueira, MG. Técnicas multivariadas de ordenação (PCA e CCA) e regressões múltiplas foram utilizadas para representar essas correlações. Altitude teve destaque como fator ambiental síntese, compreendendo vários gradientes existentes na área (em especial edáfico e de conservação) sendo, portanto, a variável que mais explicou distribuição de espécies, dinâmica florestal e estoque de carbono na área

Palavras-chave: biomassa, dinâmica florestal, distribuição de espécies, ordenação, regressão múltipla.

\section{Topographical, soil and climatic influences in an Atlantic Forest remnant in Serra da Mantiqueira, Minas Gerais State}

\begin{abstract}
Understanding the correlations among environmental variables and species distribution in a forest, including its dynamics and carbon storage, is one of the main goals in forest ecology. Therefore, the present study aimed to correlate aspects of vegetation to soil and climatic variables of a forest remnant in Bocaina de Minas, Serra da Mantiqueira, MG. Multivariate ordination techniques (PCA and CCA) and multiple regressions were used to represent these correlations. Altitude was highlighted as a synthetic environmental variable, comprising several gradients within the area (especially edaphic and conservation). This was the variable that best explained the distribution of species, forest dynamics and carbon stock in the area.
\end{abstract}

Keywords: biomass, forest dynamics, multiple regression, ordination, species distribution. 


\section{INTRODUÇÃO}

Numerosos mecanismos têm sido propostos para explicar a manutenção da alta diversidade de espécies em florestas tropicais, sendo que muitos trabalhos relacionam essa alta diversidade à também alta heterogeneidade ambiental existente nessas floretas (Rodrigues et al., 2007; Sarcinelli et al., 2012; Solórzano et al., 2012; Toledo et al., 2011b; Wright, 2002; Santos et al., 2013). A heterogeneidade ambiental pode ser resultante de fatores como variações climáticas, qualidade e intensidade de luz, regimes hídricos, topografia e propriedades físicas e químicas dos solos (Svenning, 2001) presentes nesses ambientes.

A distribuição de espécies arbóreas frequentemente se correlaciona com essas variações ambientais, sugerindo que diferenciações de nicho, ou seja, características ambientais que relacionam as espécies capazes de se estabelecer em certo local (Kneitel e Chase, 2004; Wright, 2002), podem ser importantes para manutenção da diversidade de espécies arbóreas nos trópicos. As respostas das espécies a esses fatores que interagem nas comunidades fazem com que cada local tenha características próprias e características que são comuns a outros locais, possibilitando identificar tendências (Rodrigues et al., 2007).

No entanto, diferentes padrões podem emergir em diferentes escalas. Em escala geográfica regional, a heterogeneidade florística em florestas parece estar associada a variações climáticas e altitudinais (Oliveira-Filho e Fontes, 2000). Já em escalas mais locais, as variações topográficas nas florestas tropicais podem determinar um gradiente edáfico, alterando as condições de drenagem e nutrientes no solo nesta escala (Wright, 2002), interferindo na distribuição e abundância de espécies arbóreas e na organização espacial da vegetação (Carvalho et al., 2007; Meireles et al., 2008; Phillips et al., 2004; Quesada et al., 2009; Rodrigues et al., 2007). Também aspectos de dinâmica florestal, como mortalidade têm sido relacionados e explicados por elementos do ambiente local (Phillips et al., 2004; Quesada et al., 2009; Toledo et al., 2011a; 2011b).

Além de fatores ambientais, ainda há de se considerar fatores estocásticos tais como colonização por acaso, extinção aleatória e deriva ecológica relacionados à teoria neutra (Hubbell, 2001), e a importância dos impactos advindos da fragmentação e antropização das florestas (Tabarelli et al., 2010) envolvidos e refletidos na estrutura e composição de espécies das comunidades arbóreas, especialmente na Mata Atlântica brasileira. Nesse sentido, combinar a explicação de padrões e processos ecológicos com a utilização de métodos estatísticos sofisticados e robustos corrobora o desenvolvimento de pesquisas ecológicas menos subjetivas.

As florestas nativas da Serra da Mantiqueira estão inseridas em agro-mosaicos com diferentes usos do solo, sendo atualmente a maior parte das florestas restritas a áreas em topos de morro e em altas elevações, as quais são inadequadas para a agricultura (Ribeiro e Freitas, 2010). Variações altitudinais são muito comuns nessas florestas e certamente contribuem para a heterogeneidade ambiental ao propiciar variações climáticas, edáficas e topográficas. Visando ilustrar a influência de variações na altitude, de fatores climáticos e edáficos na distribuição de espécies, no estoque de carbono e na dinâmica da vegetação de um fragmento de Floresta Ombrófila Densa, na Serra da Mantiqueira em Minas Gerais, o presente trabalho testou a seguinte hipótese: na escala de observação do estudo em questão, variáveis edáficas são, dentre as variáveis consideradas, as que mais explicam a distribuição de espécies, estoque de carbono e dinâmica da vegetação.

\section{MATERIAL E MÉTODOS}

\section{1. Área de estudo}

A área de estudo consiste em um fragmento de Floresta Ombrófila Densa de 13,71 ha 
localizado em uma sub-bacia hidrográfica próxima ao município de Bocaina de Minas, na região da Serra da Mantiqueira, sudeste de Minas Gerais, entre as coordenadas $22^{\circ} 07^{\prime} \mathrm{S}$ e $22^{\circ} 09^{\prime} \mathrm{S}$ de Latitude e $44^{\circ} 26^{\prime} \mathrm{W}$ e $44^{\circ} 29^{\prime} \mathrm{W}$ de Longitude (Figura 1), com altitudes variando aproximadamente de 1400 a 1700 metros. A região possui clima super-úmido pela classificação de Thornthwaite, ou seja, com balanço hídrico altamente positivo ao longo de todo o ano e Cwb pela classificação de Köppen, com invernos frios e menos úmidos que os verões, que são amenos e mais chuvosos (Mello et al., 2012). Consiste em uma importante região brasileira por abrigar nascentes que alimentam os rios Grande e Aiuruoca, dentre outros de menor porte. O solo dominante na microbacia é o Cambissolo Háplico Distrófico, cujo material de origem é o granito-gnaisse, com profundidade classificada com moderadamente profundo ( $<1,5 \mathrm{~m}$ ) (Menezes et al., 2009; Santos et al., 2013).

(a)

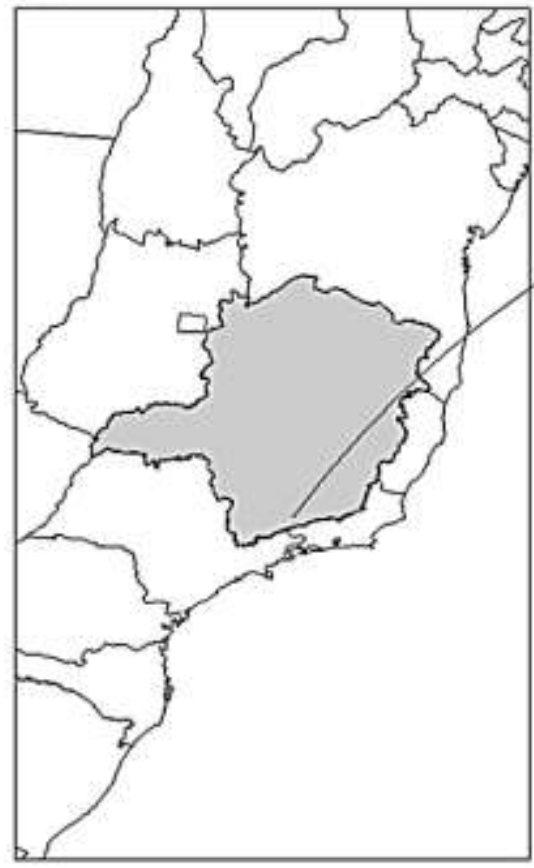

(b)

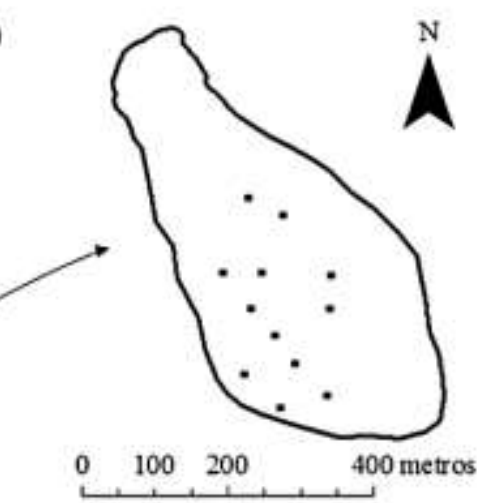

(c)

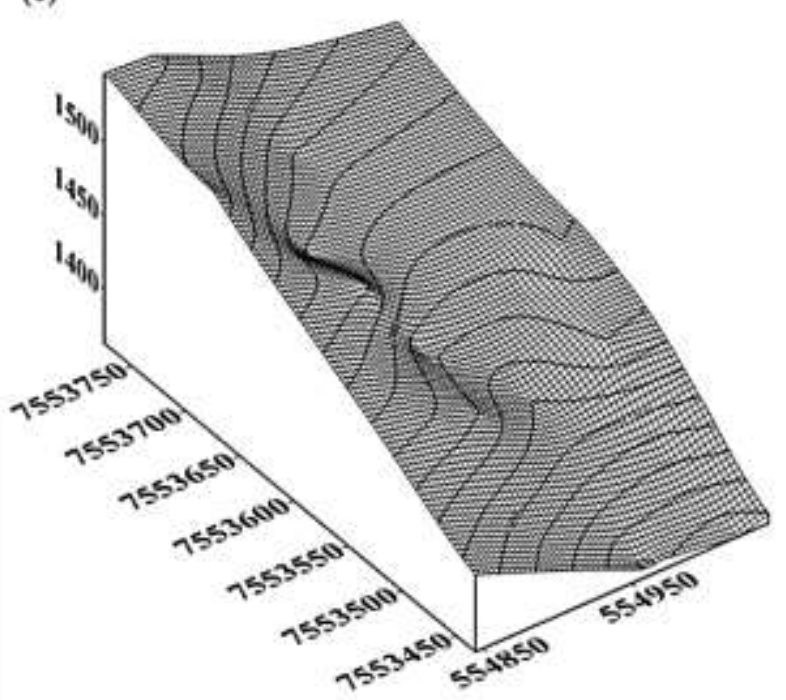

Figura 1. (a) Localização da área de estudos; (b) limites do fragmento em estudo e distribuição das parcelas amostrais utilizadas para levantamento da vegetação e variáveis ambientais; e (c) representação da inclinação da área, com suas coordenadas (latitude e longitude) em UTM, altitude em metros e isolinhas separadas em $10 \mathrm{~m}$.

\subsection{Amostragem da vegetação, estimativa do estoque de carbono, cálculo das taxas de dinâmica florestal}

A coleta dos dados da vegetação foi realizada em junho de 2009 e em julho de 2011. Nessas ocasiões, foram medidas em 12 parcelas permanentes de $400 \mathrm{~m}^{2}(20$ x $20 \mathrm{~m})$ 
distribuídas aleatoriamente na área, a altura total e a circunferência a 1,30 m do solo (CAP) de todas as árvores vivas com CAP maior ou igual a 15,7 cm. As árvores medidas foram identificadas em campo ou posteriormente por especialista.

$\mathrm{O}$ carbono presente no fuste até $3 \mathrm{~cm}$ de diâmetro da vegetação foi calculado, por parcela, para os dois inventários da floresta $(\mathrm{C} 1$ e $\mathrm{C} 2)$ por meio de equação específica para a fitofisionomia Floresta Ombrófila Densa nas sub-bacias hidrográficas do Rio Grande e Rio Piracicaba, em Minas Gerais, apresentada por Scolforo et al. (2008) (Equação 1)

$$
\mathrm{C}=\mathrm{e}^{(-11,7511954986+2,1703210582 * \ln (\mathrm{DAP})+0,945801054 * \ln (\mathrm{HT}))}
$$

em que:

C é o estoque de carbono (ton),

DAP é o Diâmetro a 1,3 m do solo $(\mathrm{cm})$,

HT é a altura total da árvore (m).

Além disso, foram calculadas as seguintes taxas de dinâmica da comunidade florestal por parcela: mortalidade (TM) $(\%)$, recrutamento (TR) $(\%)$, rotatividade em número de indivíduos $(\mathrm{RN})(\%)$, perda $(\mathrm{P})(\%)$ e ganho $(\mathrm{G})(\%)$ em área basal, rotatividade em área basal (RAB) (\%) (Oliveira-Filho et al., 1997; Sheil et al., 1995; 2000) e o ganho em carbono por parcela (GC) (ton), sendo esse último calculado por meio da diferença entre C2 (ton) e C1 (ton).

\subsection{Levantamento das variáveis ambientais}

Todas as parcelas amostrais foram georreferenciadas obtendo-se, portanto, informações de altitude (Alt) das mesmas. Além disto, uma bateria de pluviômetros foi instalada sob o dossel em pontos coincidentes com a localização das parcelas amostrais de vegetação para estudar a quantidade de chuva que efetivamente atinge a superfície do solo. A partir desses pluviômetros, obteve-se as medidas de Precipitação média mensal (Pmed) (mm), Precipitação máxima mensal (Pmax) (mm) e Precipitação mínima mensal (Pmin) (mm) para o período analisado. Amostras de solo foram coletadas nas parcelas de amostragem da vegetação para análise química e física nas profundidades: 0-20 cm, 20-50 cm e 50-100 cm, de onde foram extraídas infomações (conforme protocolo EMBRAPA, 1997) sobre teores de de teor de fósforo $(\mathrm{P})$, de alumínio ( $\mathrm{Al})$, acidez potencial $(\mathrm{H}+\mathrm{Al})$, capacidade de troca de cátios a pH 7,0 (T), saturação por bases (v), pH, soma de bases trocáveis (Sb), matéria orgânica (M.O.), densidade do solo (Dens), volume total de poros (VTP), e porcentagens de Argila, Areia e Silte no solo.

\subsection{Correlação entre distribuição de espécies e variáveis ambientais}

Devido à distribuição contínua (não abrupta) das variáveis avaliadas no ambiente, considerou-se a análise de gradientes como a abordagem mais adequada para tratar das variações das mesmas. Assim, para identificar padrões de distribuição das espécies que pudessem refletir variações ambientais, foram utilizadas análises de correspondência canônica (CCA), técnica de ordenação onde a entrada de dados é composta por duas matrizes, uma com informações de espécies e outra de variáveis ambientais (Felfili et al., 2007). No presente, caso buscando explicar a distribuição das espécies na área, relacionou-se a matriz de abundância (número de indivíduos) das espécies nas parcelas amostrais com matrizes de variáveis ambientais coletadas nessas parcelas que potencialmente explicam tal distribuição: clima e altitude; fertilidade do solo e textura do solo. Assim, foram realizadas três CCAs sendo uma com caráter climático ("CCA clima e altitude"), e duas com caráter edáfico ("CCA fertilidade do solo" e "CCA textura do solo"). 
As CCAs para distribuição das espécies foram trabalhadas da seguinte forma: no caso da "CCA clima e altitude", foram utilizadas as variáveis Alt, Pmedia, Pmax e Pmin; no caso da "CCA fertilidade do solo", foram utilizados teores médios (média das três profundidades de solo avaliadas) de $\mathrm{P}, \mathrm{Al}, \mathrm{H}+\mathrm{Al}, \mathrm{v}, \mathrm{pH}, \mathrm{Sb}$. (T) e M.O. Para a "CCA textura de solo", foram utilizados teores médios (média das três profundidades de solo avaliadas) de Dens, VTP, Argila, Areia e Silte. Para validação das análises, foram analisados em cada CCA os resultados dos p-valores do Testes de Monte Carlo para autovalores e para as correlações, ou seja, a proporção das 1000 randomizações realizadas entre espécies-ambiente com correlação maior ou igual à observada. As análises foram realizadas no software PC-ORD 5.10 (Mccune e Mefford, 2006).

\subsection{Correlação entre estoque de carbono e dinâmica da vegetação com variáveis ambientais}

Para verificar qual o fator ambiental mais explicativo do estoque de carbono da vegetação e das taxas de dinâmica, diante de muitas variáveis com potencial preditor, para evitar perda de informação no processo de modelagem e buscando evitar problemas de multicolinearidade, optou-se por fazer uma análise de componentes principais (PCA) das variáveis ambientais (Felfili et al., 2007). A PCA condensa informações contidas em um grande número de variáveis em um pequeno grupo de novas composições dimensionais, denominadas componentes. Os componentes gerados na PCA são conjuntos de variáveis independentes, não correlacionadas entre si, com distribuição normal (Felfili et al., 2007). Essa técnica foi utilizada a fim de reduzir a dimensão das três variáveis climáticas (Pmedia, Pmin e Pmax), resultando na "PCA Clima", das 8 variáveis de fertilidade (P, Al, H+Al, (T), v, $\mathrm{pH}, \mathrm{Sb}$ e M.O.), resultando na "PCA Fertilidade do solo" e cinco variáveis de textura (Dens, VTP, Argila, Areia e Silte), resultando na "PCA Textura do solo".

Assim, os dois componentes principais de cada PCA juntamente com a variável altitude (a qual foi considerada separadamente por estar correlacionada com as demais e apresentar isoladamente forte potencial preditor) foram analisadas como variáveis independentes em modelos de regressão linear múltipla para estoque de carbono e dinâmica da vegetação. Para isso, elaborou-se uma matriz de correlação de Pearson pareando os dois componentes principais de cada PCA e os seguintes dados de biomassa e dinâmica florestal: CG, TM, TR, $\mathrm{RN}, \mathrm{C} 1, \mathrm{C} 2, \mathrm{G}, \mathrm{P}$ e RAB, onde foram identificadas as maiores correlações $(>0.5$ e $<-0.5)$ que seriam utilizadas nos modelos. Os modelos gerados tiveram sua significância testada por teste $\mathrm{F}(\alpha=0,05)$ e os parâmetros associados às variáveis independentes foram testados por teste $\mathrm{t}(\alpha=0,05)$.

\section{RESULTADOS E DISCUSSÃO}

\subsection{Relações entre distribuição das espécies e variáveis ambientais}

A correlação entre abundância das espécies e as variáveis ambientais, de maneira geral, foi alta. Dentre os três fatores ambientais observados, fertilidade do solo é o que mais explica a distribuição de espécies no local, explicando 39,9\% da variação total dos dados. Clima e Altitude explicaram 31,6\% e textura do solo explicou 30,3\% (Tabela 1; Figura 2). As parcelas foram ordenadas seguindo principalmente um gradiente de altitude, acidez e argila no solo, sendo que acidez e argila parecem acompanhar o gradiente altitudinal.

A heterogeneidade ambiental tem um papel importante na diferenciação de nichos e é importante para a manutenção da diversidade de espécies arbóreas nos trópicos. Ela pode ser resultante de variações climáticas, qualidade e intensidade da radiação solar, regimes hídricos, topografia, propriedades físico-químicas dos solos e conservação e, frequentemente, determina a distribuição de espécies vegetais (Pyke et al., 2001; Zuquim et al., 2007). Em 
florestas tropicais, as variações topográficas podem determinar um gradiente edáfico, alterando as condições de drenagem e nutrientes no solo (Wright, 2002), principalmente em escala local, onde os gradientes edáfico e altitudinal formados são mais perceptíveis (Pereira et al., 2006). Padrões de distribuições das espécies refletem essas variações em florestas em escala similiar à considerada no presente estudo (França e Stehmann, 2004; Gentry, 1993; Liberman et al., 1985; Meireles et al., 2008). Em escalas maiores, trabalhos apontam flutuações no clima como responsáveis por substituição e distribuição de espécies (Pyke et al., 2001).

Tabela 1. Resumo das três análises correspondência canônica (CCAs) relacionando a abundância de espécies árbóreas em 12 parcelas permanentes em uma Floresta Ombrófila Densa em Bocaina de Minas, MG e gradientes de "Clima e Altitude", "Fertilidade do Solo" e "Textura do Solo".

\begin{tabular}{|c|c|c|c|c|c|c|}
\hline & Variáveis & Eixo 1 & Eixo 2 & Eixo 3 & p-valor & $\begin{array}{l}\text { Variância } \\
\text { total }\end{array}$ \\
\hline \multirow{3}{*}{$\begin{array}{l}\text { Clima e } \\
\text { Altitude }\end{array}$} & Autovalores & 0,484 & 0,241 & 0,205 & 0,022 & \multirow{9}{*}{2,9386} \\
\hline & $\begin{array}{c}\text { Variância acumulada para dados } \\
\text { de espécies }(\%)\end{array}$ & 16,5 & 24,7 & 31,6 & - & \\
\hline & $\begin{array}{l}\text { Correlação de Pearson ssp- } \\
\text { ambiente }\end{array}$ & 0,973 & 0,971 & 0,941 & 0,1191 & \\
\hline \multirow{3}{*}{$\begin{array}{l}\text { Fertilidade } \\
\text { do Solo }\end{array}$} & Autovalores & 0,503 & 0,353 & 0,317 & 0,2863 & \\
\hline & $\begin{array}{c}\text { Variância acumulada para dados } \\
\text { de espécies (\%) }\end{array}$ & 17,1 & 29,1 & 39,9 & - & \\
\hline & $\begin{array}{l}\text { Correlação de Pearson ssp- } \\
\text { ambiente }\end{array}$ & 0,979 & 0,981 & 0,990 & 0,4394 & \\
\hline \multirow{3}{*}{$\begin{array}{l}\text { Textura do } \\
\text { Solo }\end{array}$} & Autovalores & 0,322 & 0,297 & 0,273 & 0,9098 & \\
\hline & $\begin{array}{c}\text { Variância acumulada para dados } \\
\text { de espécies }(\%)\end{array}$ & 11,0 & 21,1 & 30,3 & - & \\
\hline & $\begin{array}{l}\text { Correlação de Pearson ssp- } \\
\text { ambiente }\end{array}$ & 0,942 & 0,956 & 0,923 & 0,6466 & \\
\hline
\end{tabular}

Nota: Eixo 1 representa o autovalor do primeiro eixo da ordenação; Eixo 2 representa o autovalor do segundo eixo da ordenação; Eixo 3 representa o autovalor do terceiro eixo da ordenação; e p-valor representa a signigicância dos autovalores e correlações de acordo com o teste de Monte Carlo.

No presente caso, a porcentagem total da variação explicada em cada agrupamento de variáveis (clima e altitude, fertilidade e textura), apontou fertilidade como o fator que mais explica a distribuição de espécies na área, em consonânsia com o que vem sendo observado por outros autores na região de estudo (Carvalho et al., 2007; Valente et al., 2011). Na área em questão, cotas mais elevadas do terreno corresponderam, de modo geral, a sítios de maior acidez (maiores teores de Al, H+Al, (T) e M.O.) e solos mais argilosos (Santos et al., 2013), o que direta ou indiretamente seleciona espécies para os diferentes locais da floresta, uma vez que cada espécie tem um intervalo de tolerância em relação às variáveis ambientais, e quase sempre os limites dessa tolerância não são bruscos em um gradiente ambiental (Rodrigues et al., 2007).

Ao mesmo tempo, a ordenação das parcelas nos diagramas de CCA apresentou uma distribuiçao condizente como o gradiente altitudinal no primeiro eixo. Assim, o solo da floresta em questão apresentou pronunciadas variações na fertilidade ao longo de um gradiente altitudinal que refletiram na distribuição e abundância das espécies. A diferença altitudinal de até $100 \mathrm{~m}$ registrada entre as parcelas do fragmento certamente contribuiu para a heterogeneidade ambiental ao propiciar a formação de gradientes topográficos e, 
consequentemente, gradientes edáficos com reflexos na composição de espécies da comunidade arbórea.

No entanto, por se tratar de um fragmento inserido numa região com forte influência antrópica, existe mais um componente atuando sobre a heterogeneidade ambiental e consequentemente a distribuição das espécies na área: os impactos. Tais impactos parecem também seguir um gradiente, sendo mais pronunciados na região mais baixa - mais acessível - e menos pronunciados nas regiões mais altas do fragmento e, apesar de não mensurados, também podem ter influência na distribuição das espécies. Fragmentos menores, como o da área estudada, são mais susceptíveis às oscilações e aos processos degradatórios decorrentes da fragmentação e antropização, principalmente pelo aumento relativo do efeito-borda (Oliveira-Filho et al., 2007), que altera a estrutura e composição das áreas afetadas. Na área, conforme se aumenta a altitude, melhor é o estado de conservação da floresta, sendo que nas áreas mais baixas, podem ser observados impactos causados por pequenas trilhas, vestígios de gados e sinais de corte seletivo.

Os impactos relatados, principalmente trilhas e cortes, desencadeiam processos iniciais de sucessão, caracterizados inicialmente pela maior disponibilidade de luz em processo semelhante ao que ocorre com clareiras. A maior disponibilidade de luz acarreta aumento no número de indivíduos e a oportunidade para espécies com alta demanda de luz observadas na área, como é o caso de Baccharis serrulata, Eremanthus erythropappus, Vismia guianensis, e Clethra scabra, e poucas espécies com tolerância à sombra, como é o caso de: Prunus myrtifolia, Toulicia subsquamulata, Ocotea corymbosa, Guapira opposita e Cybianthus detergens (Kellman et al., 1998; Oliveira-Filho et al., 2007) observadas na área mais conservada.

Portanto, dentre as variáveis quantificadas, fertilidade tem destaque na distribuição de espécies na área. No entanto, os resultados apontam uma distribuição condizente com altitude que sumariza variações edáficas e o estado de conservação não quantificado. Não se pode ignorar ainda que a distribuição de espécies em ambientes tropicais resulta da junção de dois fatores: determinístico (em decorrência das condições ambientais) e estocástico (aleatório) ocorrendo simultaneamente (Chase et al., 2011) e, na linha determinística, para caracterização mais precisa dos "habitats preferenciais" é necessário que as tendências apresentadas pelas espécies sejam observadas em outros locais.

\subsection{Relações entre estoque de carbono, dinâmica florestal e ambiente}

Dentre os três fatores ambientais analisados via PCA, fertilidade do solo foi o que apresentou um componente sumarizado com maior explicação da variação das variáveis originais $(68,76 \%)$.

Na "PCA Clima", o componente principal 1 (PC1c) explicou 50,89\% da variação total e é positivamente correlacionado com Pmin e negativamente com Pmed e Pmax ( $\mathrm{p}=0,406)$ e o componente principal 2 (PC2c) explicou $38 \%$ da variância $(\mathrm{p}=0,052)$ e foi positivamente correlacionado com Pmax e Pmin e negativamente correlacionado cm Pmed. A "PCA Fertilidade do solo" gerou o componente principal 1 (PC1f), o qual foi positivamente correlacionado com $\mathrm{P}, \mathrm{Sb}, \mathrm{v}, \mathrm{pH}$ e explicou $68,76 \%$ da variância total $(\mathrm{p}=0,001)$ e foi negativamente correlacionado com Al, H+Al, (T), M.O., e o componente principal 2 (PC2f), foi positivamente relacionado $\mathrm{com} \mathrm{pH}$ e negativamente correlacionado com as demais variáveis, explicando $17,68 \%$ da variância $(\mathrm{p}=0,987)$, evidenciando gradiente de acidez e matéria orgânica. Já a "PCA Textura do solo" gerou o componente 1 (PC1t) que explicou $62,66 \%$ da variância e foi positivamente correlacionado com VTP, Argila e Silte e negativamente correlacionado com Densidade e Areia $(\mathrm{p}=0,002)$ e o componente principal 2 
(PC2t) que explicou 25,22\% da variância e apresentou correlação positiva com Densidade e Argila e negativa com VTP, Areia e Silte $(\mathrm{p}=0,649)$, simbolizando o gradiente textural existente na área.

A matriz de correlações entre os dois componentes principais de cada PCA gerada e valores de estoque de carbono e dinâmica destacou as correlações entre altitude e as variáveis da vegetação consideradas, sendo essa variável a que mais apresentou correlação com os atributos da vegetação (Tabela 2).
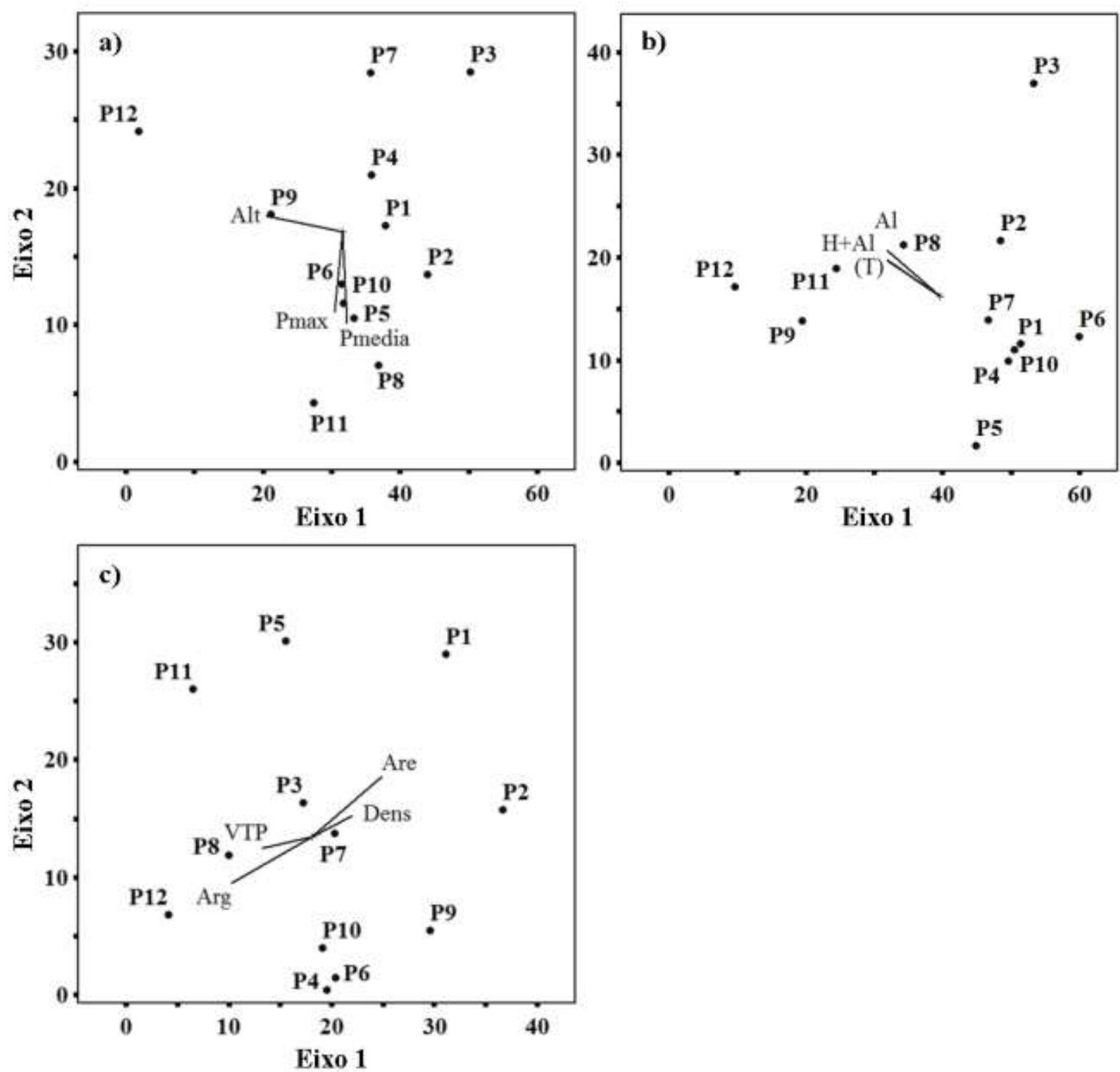

Figura 2. Diagramas produzidos pelas análises de correspondência canônica (CCAs) mostrando a ordenação de 12 parcelas permanentes (P1-P12) em uma Floresta Ombrófila Densa em Bocaina de Minas, MG em gradientes de a) "Clima e Altitude", b) "Fertilidade do Solo" e c) "Textura do Solo". 
Tabela 2. Matriz de correlação entre dois componentes principais gerados pela PCA clima (PC1c e PC2c), PCA fertilidade do solo (PCAf1 e PCAf2) e PCA textura do dolo (PCA1t e PCA2t) e os parâmetros biomassa (C1, C2 e GC) e dinâmica florestal (TM, TR, RN, P, G RAB). As correlações consideradas altas $(>0.5$ e $<-0.5)$ estão desatacadas em negrito.

\begin{tabular}{llllllll}
\hline & PC1c & PC2c & PC1f & PC2f & PC1t & PC2t & Alt \\
\hline C1 & 0,28 & $-0,09$ & $-0,45$ & $-0,34$ & $\mathbf{0 , 5 8}$ & $-0,11$ & $\mathbf{0 , 6 3}$ \\
C2 & 0,28 & $-0,02$ & $-0,48$ & $-0,39$ & $\mathbf{0 , 6 1}$ & $-0,13$ & $\mathbf{0 , 5 9}$ \\
GC & $-0,01$ & $\mathbf{0 , 7 5}$ & $-0,41$ & $\mathbf{- 0 , 6 4}$ & 0,43 & $-0,15$ & $-0,23$ \\
TM & 0,35 & $-0,09$ & 0,00 & 0,18 & $-0,30$ & 0,29 & $\mathbf{- 0 , 5 5}$ \\
TR & $-0,01$ & 0,32 & 0,37 & 0,25 & $-0,43$ & 0,07 & $\mathbf{- 0 , 7 4}$ \\
RN & 0,09 & 0,24 & 0,30 & 0,26 & $-0,43$ & 0,14 & $\mathbf{- 0 , 7 6}$ \\
P & $-0,38$ & $-0,04$ & $-0,30$ & 0,05 & 0,09 & 0,25 & $-0,20$ \\
G & $-0,10$ & 0,35 & 0,36 & 0,17 & $-0,43$ & 0,08 & $\mathbf{- 0 , 7 3}$ \\
RAB & $-0,23$ & 0,28 & 0,18 & 0,16 & $-0,31$ & 0,17 & $\mathbf{- 0 , 6 8}$ \\
\hline
\end{tabular}

Os modelos de regressão lineares apresentados (Tabela 3) correspondem aos atributos da vegetação considerados em função das variáveis que apresentaram maior correlação com os mesmos. O único modelo não significativo pelo teste $\mathrm{F}$ foi o de taxa de mortalidade, sendo os outros todos significativos e apresentando, na maioria das vezes, Altitude com variável independente com parâmetros associados significativos pelo teste t, provando mais uma vez a importância dessa variável para o conjunto de dados observados. Os coeficientes associados às variáveis independentes indicaram que altitude foi negativamente relacionada parâmetrosde dinâmica (TM, TR, RN, G e RAB) e positivamente relacionada com parametros os de biomassa (C1, C2 e GC) sugerindo uma dinâmica mais acelerada nas áreas mais baixas e com menos estoque de carbono.

No presente caso, variáveis climáticas e edáficas não foram significativas nos modelos de regressão múltipla para explicar taxas de dinâmica e biomassa, embora o gradiente de fertilidade do solo já tenha sido relatado como positivamente relacionado com taxas como a de mortalidade em outros trabalhos (Toledo et al., 2011b). Por outro lado, os modelos gerados evidenciam o papel da altitude na definição do estoque de carbono e dinâmica florestal. Novamente, altitude apresenta-se como elemento "síntese" dos outros gradientes, inclusive o gradiente de conservação observado na área.

Em termos gerais, uma dinâmica mais acelerada foi observada nas áreas mais baixas e mais impactadas do fragmento florestal. Maiores taxas de mortalidade, recrutamento e rotatividade são característicos de áreas em fase de construção da silvigênese (Hallé et al., 1978; Oldeman, 1987; 1992) após distúrbios. Nessa fase mais instável, a mortalidade se eleva em função do maior número de indivíduos e, consequentemente, maior competição dos indivíduos menores (Coomes et al., 2003), o que aumenta a rotatividade. Com a alta competição por sobrevivência, pouco é investido em crescimento individual, o que faz com que, apesar do aumento geral da área basal nessas áreas, as dimensões individuais das árvores sejam pequenas e resultem em ganhos de carbono mais baixos. A composição florística nas áreas mais impactadas também contribui para a aceleração da dinâmica, com dinâmica acelerada pela provável composição de espécies com ciclo de vida mais curto (Guimarães et al., 2008). Em setores mais altos da floresta, correspondentes a áreas mais conservadas e maduras em termos sucessionais, observam-se maiores indivíduos, com maiores estoques $\mathrm{C} 1$ e C2, menores taxas de mortalidade, recrutamento, rotatividade, ou seja, áreas mais estáveis (Felfili, 1995; Lieberman et al., 1985; Rankin-De-Merona et al., 1990; Santos et al., 1998; Swaine et al., 1987). 
Tabela 3. Resultados das regressões lineares simples e múltiplas envolvendo parâmetros biomassa (C1, C2 e GC) e dinâmica florestal florestal (TM, TR, RN, G e RAB) em função de gradientes de clima, fertilidade e textura do solo. As variáveis independentes foram selecionadas a partir da matrix de correlação (Tabela 2). Os valores p dos modelos e parâmetros significativos estão destacados em negrito.

\begin{tabular}{|c|c|c|c|c|c|c|}
\hline \multirow{3}{*}{$\begin{array}{c}\text { Variável } \\
\text { dependente }\end{array}$} & \multicolumn{3}{|c|}{ Modelos } & \multicolumn{3}{|c|}{ Medidas de ajuste } \\
\hline & \multirow{2}{*}{$\frac{\text { Intercepto }}{-}$} & \multicolumn{2}{|c|}{ Variável(is) independente(s) } & \multirow[t]{2}{*}{ p-value: $F$} & \multirow[t]{2}{*}{$\mathrm{R}^{2}$} & \multirow[t]{2}{*}{ Syx } \\
\hline & & PC1t & Alt & & & \\
\hline \multirow[t]{3}{*}{$\mathrm{C} 1$} & 0,548946 & 0,286159 & 0,016543 & 0,03155 & 0,4330 & 0,9896 \\
\hline & $(0,3781)$ & $(0,1340)$ & $(0,0811)$ & & & \\
\hline & - & PC1t & Alt & & & \\
\hline \multirow[t]{3}{*}{$\mathrm{C} 2$} & 0,808647 & 0,328390 & 0,014979 & $\mathbf{0 , 0 3 3 9 2}$ & 0,4238 & 1,019 \\
\hline & $(0,2174)$ & $(0,0997)$ & $(0,1182)$ & & & \\
\hline & - & $\mathrm{PC} 2 \mathrm{f}$ & $\mathrm{PC} 2 \mathrm{c}$ & & & \\
\hline \multirow[t]{3}{*}{$\mathrm{GC}$} & 0,22338 & $-0,04319$ & 0,06900 & 0,00395 & 0,6427 & 0,07855 \\
\hline & $(4,05 \mathrm{e}-06)$ & $(0,0672)$ & $(0,0146)$ & & & \\
\hline & - & - & Alt & & & \\
\hline \multirow[t]{3}{*}{$\mathrm{TM}$} & 0,846433 & - & $-0,008852$ & 0,06179 & 0,2372 & 0,5327 \\
\hline & $(\mathbf{0 , 0 1 8 5 )}$ & - & $(0,0618)$ & & & \\
\hline & - & - & Alt & & & \\
\hline \multirow[t]{3}{*}{$\mathrm{TR}$} & 5.11365 & - & -0.03668 & 0,00546 & 0,5100 & 1,316 \\
\hline & $(4.35 \mathrm{e}-05)$ & - & $(0.00546)$ & & & \\
\hline & - & - & Alt & & & \\
\hline \multirow[t]{3}{*}{ RN } & 2,980039 & - & $-0,022767$ & 0,00410 & 0,5359 & 0,7783 \\
\hline & $(4,94 e-05)$ & - & $(0,0041)$ & & & \\
\hline & & - & Alt & & & \\
\hline \multirow[t]{3}{*}{ G } & 9,99195 & - & $-0,05285$ & 0,00680 & 0,4894 & 1,968 \\
\hline & $(4,26 \mathrm{e}-06)$ & - & $(\mathbf{0 , 0 0 6 8 )}$ & & & \\
\hline & - & - & Alt & & & \\
\hline \multirow[t]{2}{*}{ RAB } & 5,68813 & - & $-0,02991$ & 0,01412 & 0,4149 & 1,276 \\
\hline & $(1,34 e-05)$ & - & $(0,0141)$ & & & \\
\hline
\end{tabular}

Nota: $\mathrm{R}^{2}$ representa o coeficiente de determinação e Syx representa o erro padrão residual das equações ajustadas.

Embora o crescimento de espécies vegetais seja relacionado diretamente com clima, na escala em questão, diferenças na precipitação entre as parcelas não foram suficientes para influenciar os atributos considerados. A heterogeneidade (irregularidade) do dossel, decorrente de variações em estágio sucessional, densidade, composição de espécies e até mesmo condição topográfica da floresta certamente ocasionou diferentes níveis de interceptação da precipitação no dossel da floresta sendo o efeito da precipitação efetiva possivelmente mascarado pela estrutura da floresta. 


\section{CONCLUSÃO}

A hipótese inicial do presente trabalho foi parcialmente aceita, uma vez que fertilidade do solo foi das variáveis consideradas, a que mais explicou as variações na distribuição das espécies. No entanto, as variações na fertilidade estão diretamente ligadas à condição topográfica da floresta, ou seja, às variações na altitude, a qual se mostrou o fator ambiental mais influente no estoque de carbono e dinâmica florestal no fragmento em questão.

O gradiente altitudinal foi particularmente reforçado na área por alguns impactos observados na floresta, os quais coincidem com a topografia - são mais pronunciados nas regiões mais baixas e acessíveis do fragmento florestal - e também parecem influenciar os atributos da vegetação em questão.

Assim, o presente trabalho reforça o importante papel desempenhado pela topografia, aqui representada pela variável Altitude, como "fator síntese" de uma série de variáveis que causam heterogeneidade ambiental e influenciam padrões ecológicos em escalas locais.

\section{AGRADECIMENTOS}

O presente trabalho contou com recursos do CNPq (Edital Universal 2009, Processo 471688/2009-6 - Balanço hídrico e qualidade de água em microbacia hidrográfica ocupada por Mata Atlântica na Serra da Mantiqueira, MG) e FAPEMIG (Edital Universal 2008, APQ - 00942-08 - Balanço hidrológico e produção de água numa bacia hidrográfica no ambiente da Serra da Mantiqueira, MG).

\section{REFERÊNCIAS}

CARVAlHO, W. A. C.; OLIVEIRA-FILHO, A. T.; FONTES, M. A. L.; CURI, N. Variação espacial da estrutura da comunidade arbórea de um fragmento de floresta semidecídua em Piedade do Rio Grande, MG, Brasil. Revista Brasileira de Botânica, v. 30, n. 2, p. 315-335, 2007.

CHASE, J. M.; MYERS, J. A.; B, P. T. R. S.; CHASE, J. M.; MYERS, J. A. Disentangling the importance of ecological niches from stochastic processes across scales. Philosophical Transactions of the Royal Society B, v. 366, n. 1576, p. 2351-2363, 2011. http://dx.doi.org/10.1098/rstb.2011.0063

COOMES, D. A.; DUNCAN, R. P.; ALLEN, R. B.; TRUSCOTT, J. Disturbances prevent stem size-density distributions in natural forests from following scaling relationships. Ecology Letters, v. 6, n. 11, p. 980-989, 2003. http://dx.doi.org/10.1046/j.14610248.2003.00520.x

EMPRESA BRASILEIRA DE PESQUISA AGROPECUÁRIA - EMBRAPA. Manual de métodos de análises de solo. 2. ed. Rio de Janeiro: Centro Nacional de Pesquisa de Solos, 1997. 212 p.

FELFILI, J. M. Growth, recruitment and mortality in the Gama gallery forest in central Brazil over a six-year period: 1985-1991. Journal of Tropical Ecology, v. 11, n. 1, p. 67-83, 1995. http://dx.doi.org/10.1017/S0266467400008415

FELFILI, J. M.; CARVALHO, F. A.; LIBANO, A. M.; VENTUROLI, F.; PEREIRA, B. A. S. Análise multivariada em estudos de vegetação. Brasília: UnB, 2007. 60 p. (Comunicações Técnicas Florestais, 9). 
FRANÇA, G. S.; STEHMANN, J. R. Composição florística e estrutura do componente arbóreo de uma floresta altimontana no município de Camanducaia, Minas Gerais, Brasil. Revista Brasileira de Botânica, v. 27, n. 1, p. 19-30, 2004.

GENTRY, A. H. Patterns of diversity and floristic composition in neotropical montane forests. In: GENTRY, A. H. Biodiversity and conservation of Neotropical montane forests. Bronx: The New York Botanical Garden, 1993. p. 103-126.

GUIMARÃES, J. C. C.; VAN DEN BERG, E.; CASTRO, G. C.; MACHADO, E. L. M.; OLIVEIRA-FILHO, A. T. Dinâmica do componente arbustivo-arbóreo de uma floresta de galeria aluvial no planalto de Poços de Caldas, MG, Brasil. Revista Brasileira de Botânica, v. 31, n. 4, p. 621-632, 2008.

HALlÉ, F.; OLDEMAN, R. A.; TOMLINSON, P. B. Tropical treesandforests: anarchitectural analysis. Berlin: Springer Verlag, 1978. $441 \mathrm{p}$.

HUBBELL, S. P. The unified neutral theory of biodiversity and biogeography. Princeton: Princeton University, 2001. 448 p.

KELLMAN, M.; TACKABERRY, R.; RIGG, L. Structure and function in two tropical gallery forest communities implications for forest conservation in fragmented systems. Journal of Applied Ecology, v. 35, n. 2, p. 195-206, 1998.

http://dx.doi.org/10.1046/j.1365-2664.1998.00300.x

KNEITEL, J. M.; CHASE, J. M. Trade-offs in community ecology: linking spatial scales and species coexistence. Ecology Letters, v. 7, n. 1, p. 69-80, 2004. http://dx.doi.org/10.1046/j.1461-0248.2003.00551.x

LIBERMAN, M.; LIBERMAN, D.; HARTSHORN, G. S.; PERALTA, R. Small-scale altitudinal variation in lowland wet tropical forest vegetation. The Journal of Ecology, v. 73, n. 2, p. 505-516, 1985. http://www.jstor.org/stable/2260490

MCCUNE, B.; MEFFORD, M. J. PC-ORD: multivariate analysis of ecological data. Version 5.10. Oregon: MjM Software, 2006. Software.

MEIRELES, L. D.; SHEPHERD, G. J.; KINOSHITA, L. S. Variações na composição florística e na estrutura fitossociológica de uma floresta ombrófila densa alto-montana na Serra da Mantiqueira, Monte Verde, MG. Revista Brasileira de Botânica, v. 31, n. 4, p. 559-574, 2008.

MELlO, C. R.; NORTON, L. D.; CURI, N.; YANAGI, S. N. M. Sea surface temperature (SST) and rainfall erosivity in the Upper Grande River basin, southeast Brazil. Ciência e Agrotecnologia, v. 36, n. 1, p. 53-59, 2012. http://dx.doi.org/10.1590/S141370542012000100007

MENEZES, M. D.; JUNQUEIRA JÚNIOR, J. A.; MELLO, C. R.; SILVA, A. M.; CURI, N.; MARQUES, J. J. Dinâmica hidrológica de duas nascentes, associada ao uso do solo, características pedológicas e atributos físico-hídricos na sub-bacia hidrográfica do Ribeirão Lavrinha, Serra da Mantiqueira, MG. Scientia Forestalis, v. 37, n. 82, p. 175184, 2009.

OLDEMAN, R. A. A. An architectural models, fractals and agroforestry design. In: OLDEMAN, R. A. A. Agriculture, ecosystems and environment. Amsterdam: Elsevier Science, 1992. p. 179-188. 
OLDEMAN, R. A. A. Forest ecology for silvicultural design. $3^{\text {rd }}$ ed. Wageningen: Wageningen Agricultural University, 1987. 640 p.

OLIVEIRA-FILHO, A. T.; CARVALHO, W. A. C.; MACHADO, E. L. M.; HIGUCHI, P.; APPOLINÁRIO, V.; CASTRO, G. C. et al. Dinâmica da comunidade e populações arbóreas da borda e interior de um remanescente florestal na Serra da Mantiqueira, Minas Gerais, em um intervalo de cinco anos (1999-2004). Revista Brasileira de Botânica, v. 30, n. 1, p. 149-161, 2007.

OLIVEIRA-FILHO, A. T.; FONTES, M. A. L. Patterns of floristic differentiation among Atlantic Forests in Southeastern Brazil and the Influence of Climate. Biotropica, v. 32, n. 4b, p. 793-810, 2000. http://dx.doi.org/10.1111/j.1744-7429.2000.tb00619.x

OLIVEIRA-FILHO, A. T.; MELLO, J. M.; SCOLFORO, J. R. S. Effects of past disturbance and edges on tree community structure and dynamics within a fragment of tropical semideciduous forest in southeastern Brazil over a five year period (1987-1992). Plant Ecology, v. 131, n. 1, p. 45-66, 1997. http://dx.doi.org/10.1023/A:1009744207641

PEREIRA, J. A. A.; OLIVEIRA-FILHO, A. T.; LEMOS-FILHO, J. P. Environmental heterogeneity and disturbance by humans control much of the tree species diversity of Atlantic montane forest fragments in SE Brazil. Biodiversity and Conservation, v.16, n. 6, p. 1761-1784, 2006. http://dx.doi.org/10.1007/978-1-4020-6444-9_13

PHILlIPS, O. L.; BAKER, T. R.; ARROYO, L.; HIGUCHI N.; KILLEEN, T. J.; LAURANCE, W. F. et al. Pattern and process in Amazon tree turnover, 1976-2001. Philosophical transactions of the Royal Society of London. Series B, Biological Sciences, v. 359, n. 1443, p. 381-407, 2004. http://dx.doi.org/10.1098/rstb.2003.1438

PYKE, C. R.; CONDIT, R.; AGUILAR, S.; LAO, S. Floristic composition across a climatic gradient in a neotropical lowland forest. Journal of Vegetation Science, v. 12, n. 4, p. 553-566, 2001. http://www.jstor.org/stable/3237007

QUESADA, C. A.; LLOYD, J.; SCHWARZ, M.; BAKER, T. R.; PHILLIPS, O. L.; PATINO, S. et al. Regional and large-scale patterns in Amazon forest structure and function are mediated by variations in soil physical and chemical properties. Biogeosciences, v. 6, n. 2, p. 3993-4057, 2009. http://dx.doi.org/10.5194/bgd-6-39932009

RANKIN-DE-MERONA, J. M.; HUTCHINGS, R. W.; LOVEJOY, T. E. Tree mortality and recruitment over a five-year period in undisturbed upland rain forest of the central Amazon. In: GENTRY, A. H. (Ed.). Four neotropical rainforests. London: Yale University, 1990. p. 573-584.

RIBEIRO, K. T.; FREITAS, L. Impactos potenciais das alterações no Código Florestal sobre a vegetação de campos rupestres e campos de altitude. Biota Neotropica, v. 10, n. 4, p. 239-246, 2010. http://dx.doi.org/10.1590/S1676-06032010000400029

RODRIGUES, L. A.; CARVAlHO, D. A.; OlIVEIRA-FILHO, A. T.; CURI, N. Efeitos de solos e topografia sobre a distribuição de espécies arbóreas em um fragmento de floresta estacional semidecidual, em Luminárias, MG. Revista Árvore, v. 31, n. 1, p. 25-35, 2007. http://dx.doi.org/10.1590/S0100-67622007000100004 
SANTOS, F. A. M.; PEDRONI, F.; ALVES, L. F.; SANCHEZ, M. Structure and dynamics of tree species of the Atlantic Forest. Anais da Academia Brasileira de Ciências, v. 70, n. 4, p. 873-880, 1998.

SANTOS, M. C. N. S.; MELLO, J. M.; MELLO, C. R.; AVILA, L. F. Spatial continuity of soil attributes in an Atlantic Forest remnant in the Mantiqueira Range, MG. Ciência e Agrotecnologia, v. 37, n. 1, p. 68-77, 2013. http://dx.doi.org/10.1590/S141370542013000100008

SARCINELLI, T. S.; FERNANDES-FILHO, E. I.; SCHAEFER, C. E. G. R.; MARCOJÚNIOR, P.; LEITE, F. P. Representatividade fisiográfica e pedológica de fragmentos de floresta nativa em áreas de plantios homogêneos de eucalipto. Revista Árvore, v. 36, n. 3, p. 499-509, 2012. http://dx.doi.org/10.1590/S0100-67622012000300012

SCOLFORO, J. R. S.; OLIVEIRA, A.D.; ACERBI-JÚNIOR, F. W. Inventário florestal de Minas Gerais: equações de volume, peso de matéria seca e carbono para diferentes fisionomias da flora nativa. Lavras: UFLA, 2008.

SHEIL, D.; BURSLEM, D. F. R. P.; ALDER, D. The Interpretation and misinterpretation of mortality rate measures. Journal of Ecology, v. 83, n. 2, p. 331-333, 1995. http://www.jstor.org/stable/2261571

SHEIL, D.; JENNINGS, S.; SAVILL, P. Long-term permanent plot observations of vegetation dynamics in Budongo, a Ugandan rain forest. Journal of Tropical Ecology, v. 16, n. 6, p. 765-800, 2000.

SOLÓRZANO, A.; GUEDES-BRUNI, R. R.; OLIVEIRA, R. R. Composição florística e estrutura de um trecho de floresta ombrófila densa atlântica com uso pretérito de produção de banana, no parque estadual da Pedra Branca, Rio de Janeiro, RJ. Revista Árvore, v. 36, n. 3, p. 451-462, 2012. http://dx.doi.org/10.1590/S010067622012000300007

SVENNING, J. C. On the role of microenvironmental heterogeneity in the ecology and diversification of neotropical rain-forest palms (Arecaceae). The Botanical Review, v. 67, p. 1-53, 2001. http://dx.doi.org/10.1007/BF02857848

SWAINE, M. D.; LIEBERMAN, D.; PUTZ, F. E. The dynamics of tree populations in tropical forest: a review. Journal of Tropical Ecology, v. 3, p. 359-366, 1987. http://dx.doi.org/10.1017/S0266467400002339

TABARELlI, M.; AGUIAR, A.V.; RIBEIRO, M.C.; METZGER, J.P.; PERES, C.A. Prospects for biodiversity conservation in the Atlantic Forest: lessons from aging human-modified landscapes. Biological Conservation, v. 143, n. 10, p. 2328-2340, 2010 .

TOLEDO, J. J.; MAGNUSSON, W. E.; CASTILHO, C. V.; NASCIMENTO, H. E. M. How much variation in tree mortality is predicted by soil and topography in Central Amazonia? Forest Ecology and Management, v. 262, n. 3, p. 331-338, $2011 \mathrm{a}$.

TOLEDO, M.; POORTER, L.; PENÃ-ClAROS, M; ALARCO, a.; BALCÁZAR, J., LEAÑO, C. et al. Climate is a stronger driver of tree and forest growth rates than soil and disturbance. Journal of Ecology, v. 99, n. 1, p. 254-264, 2011b. http://dx.doi.org/10.1111/j.1365-2745.2010.01741.x 
VALENTE, A. S. M.; GARCIA, P. O.; SALIMENA, F. R. G.; OLIVEIRA-FILHO, A. T. Composição, estrutura e similaridade florística da Floresta Atlântica, na Serra Negra, Rio Preto, MG. Rodriguésia, v. 62, n. 2, p. 321-340, 2011.

WRIGHT, S. J. Plant diversity in tropical forests: a review of mechanisms of species coexistence. Oecologia, v. 130, n. 1, p. 1-14, 2002. http://dx.doi.org/10.1007/s004420100809

ZUQUIM, G.; COSTA, F. R. C.; PRADO, J. Fatores que determinam a distribuição de espécies de pteridófitas da Amazônia Central. Revista Brasileira de Biociências, v. 5, n. 2, p. 360-362, 2007. 